\title{
Electromagnetic eigenmodes of spherical thin films
}

\author{
Alexander $V$. Kharlanov ${ }^{1, *}$ \\ ${ }^{1}$ Volgograd State Technical University, V. I. Lenin Avenue, 28, Volgograd, 400005, Russia
}

\begin{abstract}
Eigenmodes of the spherical and ellipsoidal dielectric thin films have been analyzed. The results of calculations of frequencies of natural electromagnetic oscillations of spherical thin films depending on their sizes and dielectric permeability of external environment are presented.
\end{abstract}

\section{Introduction}

One of the objectives of physical electronics is the investigation of physical processes in thin films, particularly, the influence of electromagnetic wave on them. Accordingly, it is useful to know the natural frequency of electromagnetic oscillations of thin films. Thin films find wide application in microelectronics. There are many types of $i_{2}$ and there are many methods of obtaining it. One of thin films is a bilayer - a double molecular layer formed by polar lipids in the aqueous medium. [1]. In lipid bilayer, molecules are oriented in such a way that their polar fragments are directed towards the aqueous phase and form two hydrophilic surfaces, and nonpolar "tails" form a hydrophobic region inside the bilayer. The bilayer is a thermodynamically advantageous form of polar lipids association in the aqueous medium. It forms a closed surface that is geometrically an ellipsoid of revolution.

The surface of the phospholipid bilayer is a complex formation. The polar heads of phospholipid molecules bordering the electrolyte form a surface layer $(0.6-1 \mathrm{~nm}$ thick) filled with electric charges and dipoles. Part of these charges and dipoles belong to the heads, the other part is made up of water molecules and electrolyte ions. Therefore, the terms «surface charges» and «surface dipoles» are largely academic. Charge and dipoles of real phospholipid surfaces are distributed in the near-surface layer. As a rule, it is considered that the thickness of this layer is negligibly small and the charges are at the boundary of the section [2].

Thus, the phospholipid bilayer can be represented as a resonator, which is a two-layer ellipsoid. To find the eigenfrequencies it is necessary to solve the Maxwell equations in spheroidal coordinates - in this case the boundary conditions will depend on only one coordinate [3].

\section{The Model}

Spheroidal coordinates are rotation-symmetric coordinate system that can be obtained by rotating around the symmetry axes of the flat elliptical coordinate system consisting of mutually orthogonal focal ellipses and hyperbols. There are 2 types of spheroidal

\footnotetext{
${ }^{*}$ Corresponding author: harlanov_av@mail.ru
} 
coordinates: rotation about the focal axis of the ellipse (the symmetry axis on which the foci are located) produces prolate spheroidal coordinates, rotation about the other axis produces oblate spheroidal coordinates. As it can be seen, in our case it is more convenient to use prolate spheroidal coordinates. It can be designated the focal length distance in the elliptical coordinate system $d$ and the axis $z$ can be considered the axis of rotation.

Prolate spheroidal coordinates are related with Cartesian (rectangular) and spherical coordinates by the following equations:

$$
\begin{gathered}
x=\frac{d}{2} \sqrt{\left(\xi^{2}-1\right)\left(1-\eta^{2}\right)} \cos \varphi, \\
y=\frac{d}{2} \sqrt{\left(\xi^{2}-1\right)\left(1-\eta^{2}\right)} \sin \varphi, \\
z=\frac{d}{2} \xi \eta, \\
r=\frac{d}{2} \sqrt{\xi^{2}+\eta^{2}-1}, \cos \theta=\frac{\xi \eta}{\sqrt{\xi^{2}+\eta^{2}-1}}, \varphi=\varphi, \\
\xi \in[1, \infty), \eta \in[-1,1], \varphi \in[0,2 \pi) .
\end{gathered}
$$

The fig. 1 shows the geometry of spheroidal thin film in prolate spheroidal coordinates. The dotted line indicates the surface section $\xi=1,5$ by the planes $x 0 y$ and $y 0 z$.

Fig. 1. Prolate spheroidal coordinates.

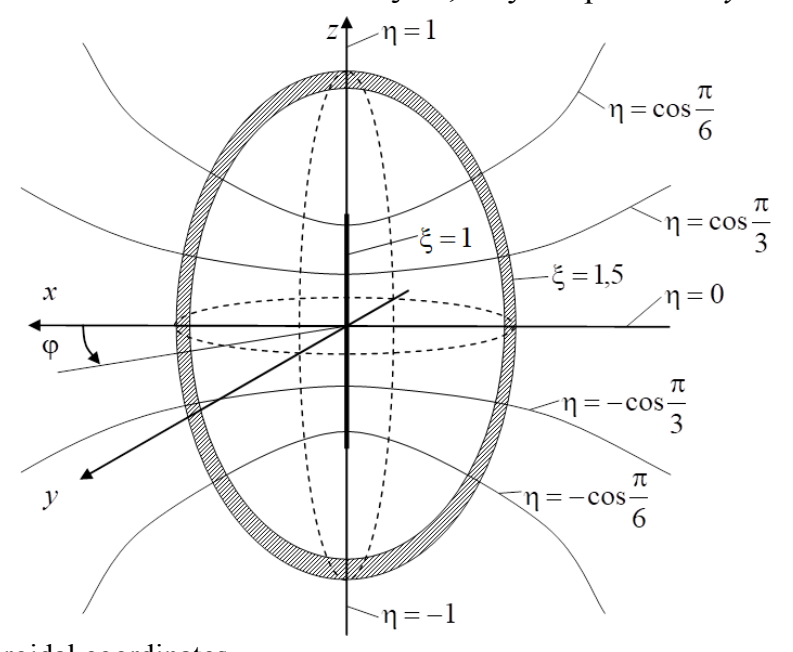

The Lamé coefficients for the prolate spheroidal coordinates are as follows:

$$
h_{\xi}=\frac{d}{2} \sqrt{\frac{\xi^{2}-\eta^{2}}{\xi^{2}-1}}, \quad h_{\eta}=\frac{d}{2} \sqrt{\frac{\xi^{2}-\eta^{2}}{1-\eta^{2}}}, \quad h_{\varphi}=\frac{d}{2} \sqrt{\left(\xi^{2}-1\right)\left(1-\eta^{2}\right)} .
$$

In a general orthogonal coordinate system, the curl is given by the expression

$$
\begin{aligned}
& \nabla \times \mathbf{A}=\frac{1}{h_{\eta} h_{\varphi}}\left(\frac{\partial}{\partial \eta} h_{\varphi} A_{\varphi}-\frac{\partial}{\partial \varphi} h_{\eta} A_{\eta}\right) \mathbf{e}_{\xi}+\frac{1}{h_{\xi} h_{\varphi}}\left(\frac{\partial}{\partial \varphi} h_{\xi} A_{\xi}-\frac{\partial}{\partial \xi} h_{\varphi} A_{\varphi}\right) \mathbf{e}_{\eta}+ \\
& +\frac{1}{h_{\xi} h_{\eta}}\left(\frac{\partial}{\partial \xi} h_{\eta} A_{\eta}-\frac{\partial}{\partial \eta} h_{\xi} A_{\xi}\right) \mathbf{e}_{\varphi},
\end{aligned}
$$


where $\mathbf{e}_{\xi}, \mathbf{e}_{\eta}, \mathbf{e}_{\varphi}$ are the coordinate unit vectors. Then the Maxwell equations for complex amplitudes of electric and magnetic field intensities

$$
\nabla \times \mathbf{E}=-i k \mathbf{H}, \nabla \times \mathbf{H}=i k \mathbf{E},
$$

where $i$ is the imaginary unit, $k$ is the wave number, can be written in the form:

$$
\begin{aligned}
& \frac{\partial}{\partial \eta} h_{\varphi} E_{\varphi}-\frac{\partial}{\partial \varphi} h_{\eta} E_{\eta}=i k h_{\eta} h_{\varphi} H_{\xi}, \frac{\partial}{\partial \eta} h_{\varphi} H_{\varphi}-\frac{\partial}{\partial \varphi} h_{\eta} H_{\eta}=-i k h_{\eta} h_{\varphi} E_{\xi}, \\
& \frac{\partial}{\partial \varphi} h_{\xi} E_{\xi}-\frac{\partial}{\partial \xi} h_{\varphi} E_{\varphi}=i k h_{\xi} h_{\varphi} H_{\eta}, \frac{\partial}{\partial \varphi} h_{\xi} H_{\xi}-\frac{\partial}{\partial \xi} h_{\varphi} H_{\varphi}=-i k h_{\xi} h_{\varphi} E_{\eta}, \\
& \frac{\partial}{\partial \xi} h_{\eta} E_{\eta}-\frac{\partial}{\partial \eta} h_{\xi} E_{\xi}=i k h_{\eta} h_{\xi} H_{\varphi}, \frac{\partial}{\partial \xi} h_{\eta} H_{\eta}-\frac{\partial}{\partial \eta} h_{\xi} H_{\xi}=-i k h_{\eta} h_{\xi} E_{\varphi} .
\end{aligned}
$$

The variables in these equations can be separated if the components $\mathbf{E}$ and $\mathbf{H}$ do not depend on the angular coordinate $\varphi$. This condition is fulfilled for the lower electrical and magnetic oscillations. With that in mind, the previous system of equations will accordingly take the form:

$$
\left\{\begin{array} { l } 
{ \frac { \partial } { \partial \eta } h _ { \varphi } E _ { \varphi } = i k h _ { \eta } h _ { \varphi } H _ { \xi } , } \\
{ \frac { \partial } { \partial \xi } h _ { \varphi } E _ { \varphi } = - i k h _ { \xi } h _ { \varphi } H _ { \eta } , } \\
{ \frac { \partial } { \partial \xi } h _ { \eta } E _ { \eta } - \frac { \partial } { \partial \eta } h _ { \xi } E _ { \xi } = i k h _ { \eta } h _ { \xi } H _ { \varphi } , }
\end{array} \quad \left\{\begin{array}{l}
\frac{\partial}{\partial \eta} h_{\varphi} H_{\varphi}=-i k h_{\eta} h_{\varphi} E_{\xi}, \\
\frac{\partial}{\partial \xi} h_{\varphi} H_{\varphi}=i k h_{\xi} h_{\varphi} E_{\eta}, \\
\frac{\partial}{\partial \xi} h_{\eta} H_{\eta}-\frac{\partial}{\partial \eta} h_{\xi} H_{\xi}=-i k h_{\eta} h_{\xi} E_{\varphi} .
\end{array}\right.\right.
$$

It is possible to separate variables using scalar functions $P(\xi, \eta)$ and $Q(\xi, \eta)$, known as Abraham's potentials:

$$
P=h_{\varphi} E_{\varphi}, Q=h_{\varphi} H_{\varphi} .
$$

From the second, fourth and fifth equations of the system (2) it is possible to obtain:

$$
\frac{\partial}{\partial \xi} \frac{h_{\eta}^{2}}{h_{\xi} h_{\eta} h_{\varphi}} \frac{\partial Q}{\partial \xi}+\frac{\partial}{\partial \eta} \frac{h_{\xi}^{2}}{h_{\xi} h_{\eta} h_{\varphi}} \frac{\partial Q}{\partial \eta}+k^{2} \frac{h_{\xi} h_{\eta} h_{\varphi}}{h_{\varphi}^{2}} Q=0 .
$$

Or using the scale factors (1):

$$
\left(\xi^{2}-1\right) \frac{\partial^{2} Q}{\partial \xi^{2}}+\left(1-\eta^{2}\right) \frac{\partial^{2} Q}{\partial \eta^{2}}+c^{2}\left(\xi^{2}-\eta^{2}\right) Q=0,
$$

where $c=k d / 2$. Similar equations can be obtained for the potential $P$. Separating the variables in the last equation by presenting the potential $Q$ in the form of $Q(\xi, \eta)=U(\xi) V(\eta)$, the system of equations is obtained:

$$
\left\{\begin{array}{l}
\left(\xi^{2}-1\right) \frac{d^{2} U}{d \xi^{2}}+\left(-\lambda+c^{2}\left(\xi^{2}-1\right)\right) U=0 \\
\left(1-\eta^{2}\right) \frac{d^{2} V}{d \eta^{2}}+\left(\lambda+c^{2}\left(1-\eta^{2}\right)\right) Q=0
\end{array}\right.
$$


where $\lambda$ is the separation constant, $U=E_{\varphi} \sqrt{\xi^{2}-1}$, and $V=\frac{E_{\varphi} d}{2} \sqrt{1-\eta^{2}}$. To find the eigenfrequencies of electromagnetic oscillations of the ellipsoidal dielectric electromagnetic resonator, it is necessary to know the dependence of the fields on the coordinate, i.e. the solution of the first equation of the system (3). Substituting $U$ in an explicit form in this equation the following expression can be obtained:

$$
\begin{aligned}
& \left(\xi^{2}-1\right) \frac{d^{2}}{d \xi^{2}}\left(E_{\varphi} \sqrt{\xi^{2}-1}\right)+\left(-\lambda+c^{2}\left(\xi^{2}-1\right)\right)\left(E_{\varphi} \sqrt{\xi^{2}-1}\right)=0 \Rightarrow \\
& \frac{d}{d \xi}\left(\xi^{2}-1\right) \frac{d E_{\varphi}}{d \xi}+\left(-\lambda+c^{2}\left(\xi^{2}-1\right)-\frac{1}{\xi^{2}-1}\right) E_{\varphi}=0 .
\end{aligned}
$$

The solution of this differential equation is the prolate radial spheroidal wave functions of the first and second order $R_{1 l}(c, \xi)$ and $R_{1 l}^{(2)}(c, \xi)$ [4-6]. Similarly, it can be shown that the solution of the second equation of the system (3) is the angular spheroidal wave functions $S_{1 l}(c, \xi)$, which are calculated using the Legendre polynomials.

The solution for magnetic oscillations can be written as follows:

$$
\begin{gathered}
E_{\xi}=0, \\
E_{\eta}=-i \sqrt{\frac{\mu_{p j} \mu_{0}}{\varepsilon_{p j} \varepsilon_{0}}} \frac{k l \sqrt{\varepsilon_{p j}}}{\xi \eta}\left[A_{j} R_{1 l}\left(k \frac{d_{j}}{2} \sqrt{\varepsilon_{p_{j}}}, \xi\right)+B_{j} R_{1 l}^{(2)}\left(k \frac{d_{j}}{2} \sqrt{\varepsilon_{p} j}, \xi\right)\right] P_{1}^{l}(\eta) e^{i \omega t}, \\
E_{\varphi}=i \sqrt{\frac{\mu_{p j} \mu_{0}}{\varepsilon_{p j} \varepsilon_{0}}} \frac{k l \sqrt{\varepsilon_{p j}}}{\xi}\left[A_{j} R_{1 l}\left(k \frac{d_{j}}{2} \sqrt{\varepsilon_{p_{j}}}, \xi\right)+B_{j} R_{1 l}^{(2)}\left(k \frac{d_{j}}{2} \sqrt{\varepsilon_{p} j}, \xi\right)\right] \frac{d P_{1}^{l}(\eta)}{d \eta} e^{i \omega t}, \\
H_{\xi}=\frac{2}{\xi^{2}}\left[A_{j} R_{1 l}\left(k \frac{d_{j}}{2} \sqrt{\varepsilon_{p_{j}}}, \xi\right)+B_{j} R_{1 l}^{(2)}\left(k \frac{d_{j}}{2} \sqrt{\varepsilon_{p j}}, \xi\right)\right] P_{1}^{l}(\eta) e^{i \omega t}, \\
H_{\eta}=\frac{1}{\xi} \frac{d\left(\left[A_{j} R_{1 l}\left(k \frac{d_{j}}{2} \sqrt{\varepsilon_{p_{j}}}, \xi\right)+B_{j} R_{1 l}^{(2)}\left(k \frac{d_{j}}{2} \sqrt{\varepsilon_{p} j}, \xi\right)\right]\right)}{d \xi} \frac{d\left(P_{1}^{l}(\eta)\right)}{d \eta} e^{i \omega t}, \\
H_{\varphi}=\frac{l}{\xi \eta} \frac{d\left(\left[A_{j} R_{1 l}\left(k \frac{d_{j}}{2} \sqrt{\varepsilon_{p_{j}}}, \xi\right)+B_{j} R_{1 l}^{(2)}\left(k \frac{d_{j}}{2} \sqrt{\varepsilon_{p} j}, \xi\right)\right]\right)}{d \xi} P_{1}^{l}(\eta) e^{i \omega t},
\end{gathered}
$$

where $k$ is the wave number in free space, $j=1$ for inner space, $j=2$ for thin film, $j=3$ for outer space, $B_{1}=0, A_{4}=0, \varepsilon_{p j}$ is the dielectric permittivity of $\mathrm{j}$-th material of the resonator, $\omega$ is the frequency of oscillations, $l=1,2,3, \ldots$.

Since the film surface has charges, it can be considered as a closed resonator in the first approximation, i.e. it can be considered only as a closed resonator, i.e. it is possible to consider only the solutions for the 1 st and 2 nd regions and to apply the boundary conditions "metal-dielectric": near the surface of the metal the tangential component of the electric field and the normal component of the magnetic field will be equal to zero.

\section{Results and Discussion}

For the calculation the following parameters are taken: thickness of bilayer is $10 \mathrm{~nm}$, ellipsoidal dimensions $-1-1000$ microns, dielectric permittivity of layer $\left(\varepsilon_{2}\right)$ is 2 , dielectric permittivity of water $\left(\varepsilon_{1}, \varepsilon_{3}\right)$ is calculated according to Debai's theory $[7,8]-$ see fig. 2 . 


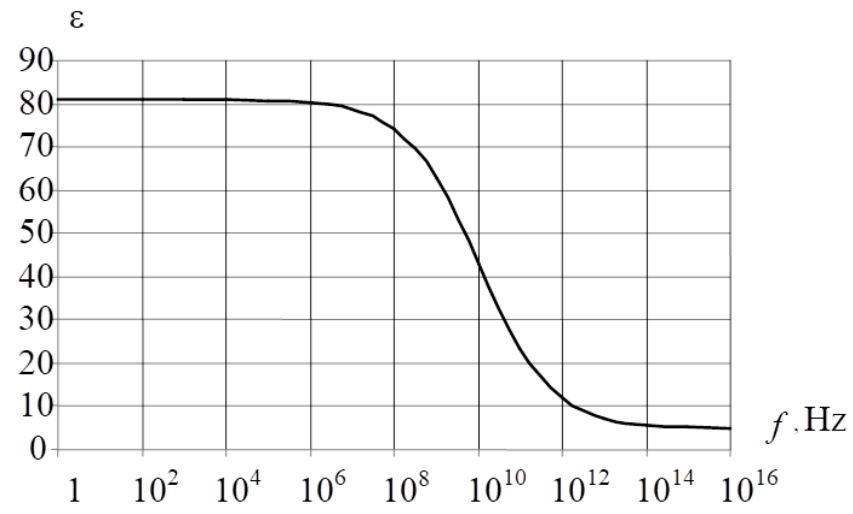

Fig. 2. Frequency dependence of the dielectric constant of water.

For the calculation of the frequencies of the inner part of the ellipsoid, the semi-axes of the ellipse $(a, b)$ are introduced, with $d / 2=\sqrt{b^{2}-a^{2}}$. Figure 3 shows the dependence of the $H_{101}$ and $E_{101}$ oscillation frequencies on the form of the resonator $(b=10 \mu \mathrm{m})$. Similar results were obtained in [9].

$$
f, 10^{13} \mathrm{~Hz}
$$

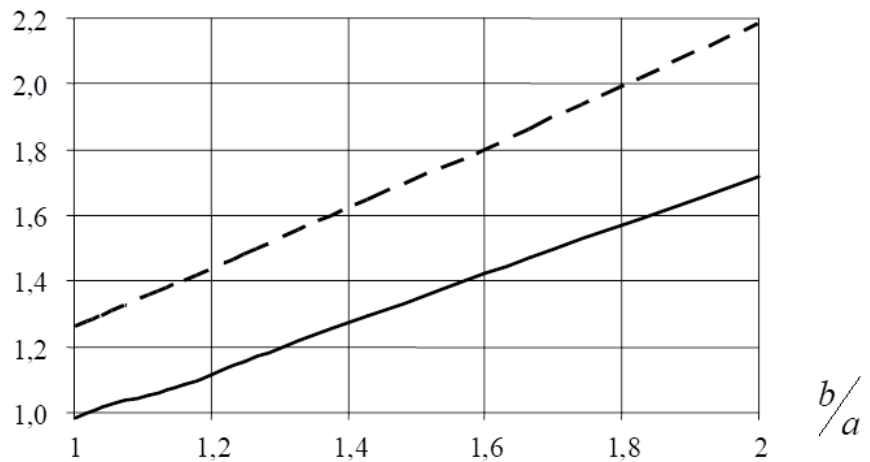

Fig. 3. Dependence of oscillation frequency on the shape of the resonator $-H_{101},---E_{101}$.

As it can be seen, the frequency changes proportionally to the ratio of half-axes and at a slight difference of the form of the resonator from the sphere $(a \approx b)$ the frequencies will also slightly differ from the eigenmods of the spherical resonator. The presented calculations are made for prolate spheroidal coordinates, similar results are obtained for oblate spheroidal coordinates. Since small deviations from sphericity do not significantly change the eigenfrequencies, it is possible to apply the spherical functions of Bessel and Neumann $\left(j_{n}(x)\right.$ and $\left.y_{n}(x)\right)$ instead of radial spheroidal functions $R_{1 l}(c, \xi)$ and $R_{1 l}^{(2)}(c, \xi)$.

From the condition of continuity of fields on the boundary of the media interface, it is possible to get the equation for the calculation of wave numbers [10]:

$$
\begin{aligned}
& {\left[j_{n}\left(x_{1}\right) \frac{d}{d x_{2}}\left(x_{2} j_{n}\left(x_{2}\right)\right)-j_{n}\left(x_{2}\right) \frac{d}{d x_{1}}\left(x_{1} j_{n}\left(x_{1}\right)\right)\right]\left(y_{n}\left(x_{4}\right) x_{3} y_{n-1}\left(x_{3}\right)-y_{n-1}\left(x_{4}\right) x_{4} y_{n}\left(x_{3}\right)\right)=} \\
& =\left[j_{n}\left(x_{1}\right) \frac{d}{d x_{2}}\left(x_{2} y_{n}\left(x_{2}\right)\right)-y_{n}\left(x_{2}\right) \frac{d}{d x_{1}}\left(x_{1} j_{n}\left(x_{1}\right)\right)\right]\left(y_{n}\left(x_{4}\right) x_{3} j_{n-1}\left(x_{3}\right)-y_{n-1}\left(x_{4}\right) x_{4} j_{n}\left(x_{3}\right)\right),
\end{aligned}
$$


where $x_{1}=k_{1} R_{1}, x_{2}=k_{2} R_{1}, x_{3}=k_{2} R_{2}, x_{4}=k_{3} R_{2}, k_{1}, k_{2}, k_{3}$, - wave numbers for the inner space, thin layer and outer space, respectively, $R_{1}$ and $R_{2}$ - inner and outer radii of the thin layer. Figure 4 shows the dependence of the lower frequencies of magnetic and electrical oscillations for dielectric and closed resonators on its size.

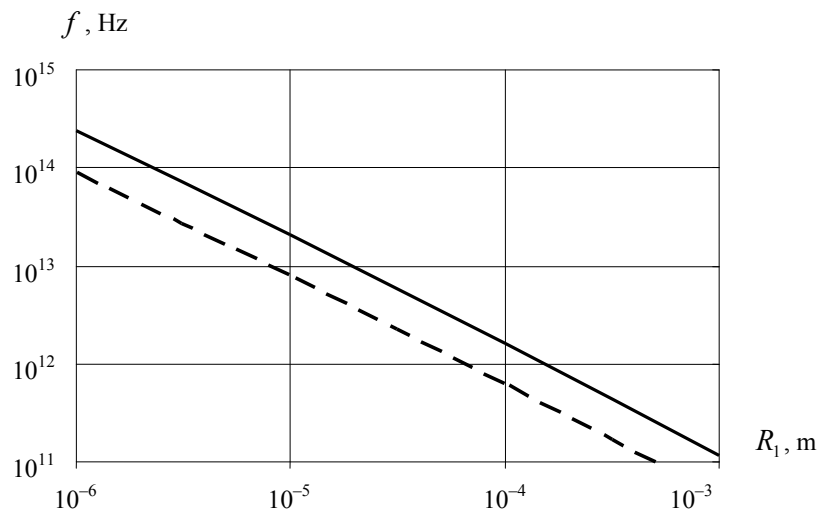

Fig. 4. Dependence of oscillation frequency on resonator radius. $-H_{101},-\cdot-E_{101}$ for the dielectric resonator.

Interesting results can be obtained by finding the dependence of natural frequencies on dielectric permittivity of the external medium. It is possible because thin lipid bilayer is a border between inner and outer media. When water is diluted with another liquid, the dielectric permittivity changes, and there is no change in the area limited by the bilayer.

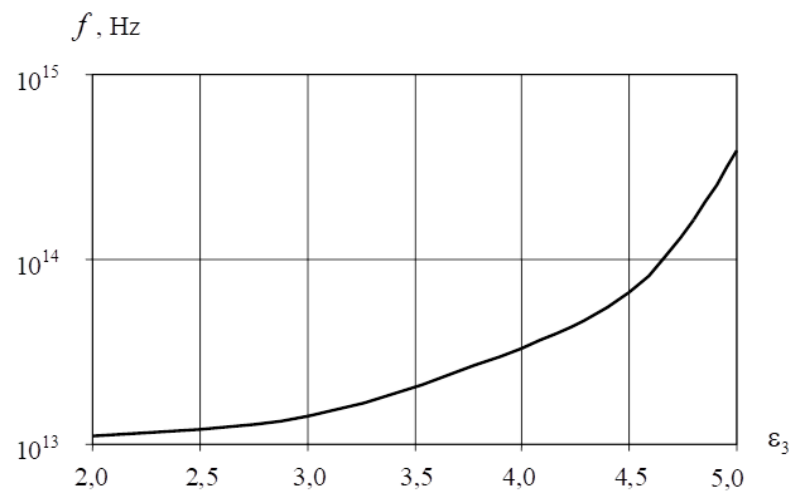

Fig. 5. Frequency dependence of oscillation on the dielectric constant of the external medium.

To calculate the frequencies (see figure 5), the following parameters were taken: the radius of the resonator $R_{1}=10 \mu \mathrm{m}$, the thickness of the thin film $R_{2}-R_{1}=10 \mathrm{~nm}$, the relative permittivity of the inner space, limited by the film $\varepsilon_{1}=5$. It can be seen that the natural frequency of oscillations is highly dependent on the dielectric permittivity of the areas separated by a thin film and will be maximum when they are equal.

Thus, it can be concluded that the natural frequencies of electromagnetic oscillations are mainly in the terahertz range, very promising at the present stage of electronics development. The strong correlation between natural frequencies and the dielectric permittivity of the medium can be used to control and measure the concentration of various substances dissolved in water. 


\section{References}

1. V. Kalyuzhny, Dictionary of nanotechnological and nanotechnology-related terms (Fizmatlit, Moscow, 2010)

2. B. V. Deryagin, N. V. Churaev, F. D. Ovcharenko, Water in dispersed systems (Chemistry, Moscow, 1989)

3. L. A. Weinstein, Open resonators and open waveguides (Sov. radio, Moscow, 1966)

4. I. V. Komarov, Ponomarev L. I., Slavyanov S. Y. Spheroidal and Coulomb spheroidal functions (Science, Moscow, 1976)

5. M. M. Abramovits, I. Stigan, Handbook on special functions (Science, Moscow, 1979)

6. K. Flammer, Tables of wave spheroidal functions (BMT; Issue 17, 1962)

7. I. S. Reza, Y. M. Poplavko, Dielectrics. Main properties and applications in electronics (Radio and communication, Moscow, 1989)

8. V. G. Artemov, A. A. Volkov, Water and Ice Dielectric Spectra Scaling at $0^{\circ} \mathrm{C}$, Ferroelectrics, 466:1, pp. 158 - 165, (2014)

9. M. Cifra, Electrodynamic eigenmodes in cellular morphology, BioSystems, v. 109(3), p. 356-366 (2012)

10. M.E. Ilchenko, Dielectric resonators (Radio and communication, Moscow, 1989) 